\title{
Inflammatory fibroid polyp (Vanek's tumour) of the ileum causing ileocolic intussusception
}

Kokulan Selvarajah ${ }^{1}$, Krishnamoorthy Ananthamoorthy ${ }^{1}$, Nilesh Fernandopulle ${ }^{2}$, S. T Sarma ${ }^{3}$, Niluka Ranathunga ${ }^{4}$

'Postgraduate Institute of Medicine, Colombo

${ }^{2}$ Department of Surgery, Faculty of Medicine, Colombo

${ }^{3}$ Department of Surgery, Faculty of Medicine, Jaffna

${ }^{4}$ Teaching Hospital, Jaffna

Keywords: Inflammatory fibroid polyp; adult intussusception; ileocolic intussusception; Vanek's tumour, PDGFR $\alpha$ mutation

\section{Introduction}

Inflammatory fibroid polyp (IFP) is a rare reactive, benign gastrointestinal tract lesion, affecting, mainly elderly population. Its clinical, endoscopic and radiological pictures may mimic that of a malignancy and can give rise to a diagnostic dilemma. Patients with IFP may present with intussusception, intestinal obstruction or haematochezia. Treatment options include surgery and in limited cases endoscopic resection.

\section{Case Report}

A 55-year-old Sri Lankan Tamil female presented to the surgical casualty ward with a history of acute onset abdominal pain, constipation and abdominal distention for 2 days. Her past medical and surgical histories were unremarkable. She did not have a significant family history of gastrointestinal tract malignancies. On examination, she was pale, dehydrated, and her abdomen was tender and distended with no palpable masses. Per rectal examination was unremarkable. Initial lab works were unremarkable except a mild anaemia (11.2 g/dL). Contrast-enhanced CT of the abdomen revealed an ileocolic intussusception but did not show the leading cause. Her colonoscopy revealed a pedunculated growth at the hepatic flexure region, and biopsies were taken. Biopsies showed only inflammatory changes without any specific diagnosis. Subsequently, she underwent an exploratory laparotomy and found to have a growth at the ileal region causing an ileocolic intussusception. The lesion with a segment of bowel was resected en bloc followed by an end to end primary anastomosis. Gross pathological examination showed a $40 \mathrm{~mm}$ size, grey, firm, pedunculated, lesion arising from the submucosal layer with a homogeneous pale white cut surface. There were no haemorrhagic or necrotic areas. Rest of the ileum and colon

Correspondence: Kokulan Selvarajah

E-mail: selvarajah.kokulan@gmail.com

Received: 08-10-2018 Accepted: 25-10-2018

iD http://http://orcid.org/0000-0003-3962-063X DOI: http://doi.org/10.4038/sljs.v36i3.8536

The Sri Lanka Journal of Surgery 2018; 36(3): 30-31 were macroscopically unremarkable. Microscopic examinations revealed a well-circumscribed lesion with numerous dilated thin walled vessels and spindle cells in an oedematous stroma without any malignant features. She recovered from the surgery without complications and was discharged in three days.

\section{Discussion}

Inflammatory fibroid poly (IFP) is a rare gastrointestinal tract reactive lesion. The first case was reported by Vanek in 1949, but the name was proposed by Helwig and Ranier in 1953. IFP has been traditionally described as a non-neoplastic lesion, but recent molecular studies show activating mutations of platelet-derived growth factor receptor $\alpha$ (PDGFR $\alpha$ ) in $70 \%$ of the cases, suggesting a possible neoplastic pathology [1]. IFP affects all the age groups without a sex preference. Majority of cases have been reported in the elderly population with a peak incidence in fifth to seventh decades [2].

Approximately $90 \%$ of cases of intussusception in adults are secondary to a pathologic condition that serves as a lead point for the intussusception, such as carcinomas, Meckel's diverticulum or rarely IFP. Patients with IFP may also present with intestinal obstruction and haematochezia. IFP patients presenting with lower urinary tract symptoms are also reported. IFPs can arise from any part of the gastrointestinal tract, but gastric antrum is the commonest site, accounting for $70 \%$ of the cases followed by small intestine in $20 \%$. It rarely affects the oesophagus and colorectum [3]. There are cases of IFP affecting gallbladder reported as well [1]. Endoscopically approximately $70 \%$ of the IFPs appear semi-pedunculated and the rest are sessile, arising from the submucosa covered by mucosa. Occasionally the polyp can invade the mucosa or extend into the muscularis propria. Most of the IFPs measure less than $5 \mathrm{~cm}$ in diameter, although IFPs up to $20 \mathrm{~cm}$ are reported [4].

Contrast-enhanced CT is sensitive in diagnosing intussusception, showing an outer intussuscipiens and inner intussusceptum, giving a sausage-shaped mass appearance in its longitudinal axis and as a target lesion in cross section. Even though CT is sensitive in diagnosing the intussusception, it fails to identify the cause as IFP in most of the 
instances [4].

The reported incidence of malignancy in ileocolic and colocolic intussusception ranges between 43 and $100 \%$. Since the preoperative investigations fail to identify the cause in a majority of the cases, surgical intervention is needed as diagnosis and treatment [4]. There is an argument whether to proceed with an en bloc resection or a more limited resection after reduction. Reduction before resection may allow a more limited resection; but manipulation itself increases the risk of intraluminal seeding or tumour dissemination of a malignant lesion [5]. In cases of small intestinal intussusception, malignancy found to be the cause in up to $47 \%$, and most lesions are metastatic [4]. Therefore, it is recommended to reduce the intussusception before a limited resection [5[. However, in ileocolic and colo-colic intussusception the possibility of a malignancy ranges from $43-100 \%$ with the majority of the lesions being primary rather than metastatic. Therefore, it is recommended to resect the lesion en bloc without reduction $[4,5]$. It can be approached by open method via a midline laparotomy as in this case report. Laparoscopy has been shown to be an equally effective alternative with manual reduction done after extra corporealization [2].

Even though endoscopic resection is a justifiable treatment approach due to the benign nature of IFP, use of therapeutic endoscopy for IFP is rare due to the size of the lesion, failure of preoperative confirmation of the diagnosis, and the urgency of symptoms [3].

Microscopically the IFP comprises of numerous small vessels, fibroblasts in an oedematous stroma, infiltrated by inflammatory cells mainly eosinophils and plasma cells [3]. The fibroblasts are arranged in a concentric "onion-skin" pattern centered on blood vessels giving rise to frequently seen whorled appearance. In immunohistochemical stains, IFPs express positivity to CD34, S100, Fascin and CD35 and negativity to CD117, Bcl-2 and c-kit. Positivity to CD35 and Fascin has been presented as evidence of dendritic cell origin. Negativity to CD117, Bcl-2 and c-kit differentiate IFPs from Gastrointestinal Stromal Tumours (GIST) [3].

All authors disclose no conflict of interest. The study was conducted in accordance with the ethical standards of the relevant institutional or national ethics committee and the Helsinki Declaration of 1975, as revised in 2000 .

\section{Reference}

1. Martini M, Santoro L, Familiari P, Costamagna G, Ricci R. Inflammatory fibroid polyp of the gallbladder bearing a: Platelet-Derived Growth Factor Receptor Alpha mutation. Arch Pathol Lab Med. 2013;137(5):721-724. doi:10.5858/arpa.20120218-CR

2. Abboud B. Vanek's tumor of the small bowel in adults. World J Gastroenterol. 2015;21(16):4802-4808. doi:10.3748/wjg.v21.i16.4802

3. Ihimoyan A, Chelimilla H, Balar B. An inflammatory fibroid polyp in the hepatic flexure of the colon treated with argon plasma coagulation, endoscopic clipping and polypectomy. Case Rep Gastroenterol. 2012;6(1):63-68. doi:10.1159/000336401

4. Akbulut S. Intussusception due to inflammatory fibroid polyp: A case report and comprehensive literature review. World J Gastroenterol. 2012;18(40):5745-5752. doi:10.3748/wjg.v18.i40.5745

5. Sarma D, Prabhu R, Rodrigues G. Adult intussusception: A sixyear experience at a single center. Ann Gastroenterol. 2012;25(2):128-132.

\section{Learning Points:}

- IFP is one of the rare causes of adult intussusception, which may mimic malignancy.

- Preoperative diagnosis is difficult, and hence warrants surgical intervention for both diagnosis and treatment.

- Colonic intussusception, in general, should not be manually reduced to reduce the potential risk of malignant venous dissemination.

- Endoscopy plays a limited role in terms of definitive management. 